\title{
Immunotherapy for esophageal carcinoma: a narrative review
}

\author{
Takahiro Miyamoto ${ }^{1}$, Ken Kato ${ }^{2}$ \\ ${ }^{1}$ Department of Chemotherapy Center, Osaka Medical College, Osaka, Japan; ${ }^{2}$ Department of Head and Neck Medical Oncology, National Cancer \\ Center Hospital, Tokyo, Japan \\ Contributions: (I) Conception and design: K Kato; (II) Administrative support: T Miyamoto; (III) Provision of study materials or patients: All authors; \\ (IV) Collection and assembly of data: All authors; (V) Data analysis and interpretation: All authors; (VI) Manuscript writing: All authors; (VII) Final \\ approval of manuscript: All authors. \\ Correspondence to: Ken Kato, MD, PhD. Department of Head and Neck Medical Oncology, National Cancer Center Hospital, 5-1-1 Tsukiji, Chuo- \\ ku, Tokyo 104-0045, Japan. Email: kenkato@ncc.go.jp.
}

\begin{abstract}
The main purpose of systemic treatment for advanced esophageal squamous cell carcinoma (ESCC) is to alleviate symptoms and extend the survival period. This systemic treatment is mainly composed of cytotoxic drugs. However, based on the results of phase 3 trials examining programmed cell death 1 (PD-1) inhibitors, such as nivolumab and pembrolizumab, in ESCC, PD-1 inhibitors are expected to become the standard therapy for previously treated ESCC patients. Immunotherapy, as combination therapy, including dual immune checkpoint inhibitors and combination therapy with cytotoxic drugs, is under investigation. This review examines the existing clinical data and describes the future of systemic treatment of advanced ESCC.
\end{abstract}

Keywords: Esophageal cancer; immune checkpoint inhibitors; clinical trials; combination therapy

Received: 08 May 2020; Accepted: 27 July 2020; Published: 10 July 2021.

doi: $10.21037 /$ shc-20-69

View this article at: http://dx.doi.org/10.21037/shc-20-69

\section{Introduction}

Esophageal cancer is a concern worldwide, with 572,000 new cases and 509,000 deaths each year, and the incidence and mortality are gradually increasing (1). Esophageal cancer is the seventh most commonly diagnosed cancer worldwide and the sixth most common cause of cancerrelated death (1). Esophageal cancer is very aggressive; most patients are diagnosed at advanced stages and have a poor prognosis. Esophageal cancer has two main histological subtypes: squamous cell carcinoma (SCC) and adenocarcinoma (AC). Their etiologies and prognoses vary, with the prognosis of SCC being worse than that of AC (2). Therefore, these two histologic subtypes should be considered different diseases that require different treatment strategies.

For a long time, only cytotoxic agents were effective for esophageal squamous cell carcinoma (ESCC) and AC, but the results of recent clinical trials have shown the effectiveness of immunotherapy. This review outlines the use of immunotherapy for esophageal cancer.

We present the following article in accordance with the Narrative Review reporting checklist (available at http:// dx.doi.org/10.21037/shc-20-69).

\section{Method}

For this narrative review, research has been conducted about immune checkpoint inhibitors, immune-related adverse events (irAEs), and predictive biomarkers for esophageal carcinoma. The research was limited to papers published between 2007 and 2020. Clinical trials and guidelines related to immunotherapy for esophageal carcinoma were analyzed. A research strategy was undertaken using databases such as Medline, Ovid, EMBASE, Cochrane, and ClinicalTrials.gov.

\section{Immune checkpoint inhibitors in clinical trials}

Inhibitory immune checkpoint molecules [e.g., programmed 
cell death 1 (PD-1), cytotoxic T lymphocyte-associated antigen 4 (CTLA-4), and lymphocyte activation gene3] and stimulatory immune checkpoint molecules (e.g., CD40 ligand, OX40, and inducible T-cell costimulatory molecule) play a role in the maintenance of immunological homeostasis. Dysregulation of inhibitory immune checkpoint molecule expression by cancer cells is associated with escaping immune surveillance, a key mechanism driving tumor progression. PD-1 is an immunosuppressive receptor highly expressed on immune cells such as activated T cells, B cells, and NK cells (3). Interaction between PD-1 and programmed cell death ligand (PD-L) 1 or 2 can mediate suppression of $T$ cell activity through the negative regulation of $\mathrm{T}$ cell receptor and CD28 signaling (3). PDL1 overexpression is prevalent in many cell types, including antigen-presenting cells (4). The upregulation of PD-L1 is also found in many types of cancer, suggesting that the PD-1/PD-L1 pathway impairs antitumor response (3). PDL1 overexpression is observed in $18.4-82.8 \%$ of ESCCs, and these patients have decreased survival (5).

Immune checkpoint inhibitors targeting the PD-1/PDL1 pathway have revolutionized the therapeutic landscape of many different cancers. Nivolumab, a human IgG4 monoclonal antibody against PD-1, has been approved by the US Food and Drug Administration for patients with non-small cell lung cancer, melanoma, and renal cell carcinoma $(6,7)$. For ESCC, a multicenter phase 2 study evaluated the activity of nivolumab after the failure of fluoropyrimidine-based, platinum-based, and taxanebased chemotherapy without patient selection based on PD-L1 expression in tumors (ONO-4538-07) (8) and found a promising antitumor effect of nivolumab, with a $17 \%$ objective response rate. The median progressionfree survival (PFS) was short (1.5 months), but the median overall survival (OS) was 10.8 months. Therefore, some patients have achieved a durable response. Treatmentrelated adverse events were similar to those in previous studies examining nivolumab monotherapy in other types of tumors. A randomized phase 3 trial (ONO-4538-24/ CA209-473_ATTRACTION-3 trial) was performed to compare nivolumab versus docetaxel or paclitaxel in patients with ESCC refractory to fluoropyrimidine and platinum (NCT02569242). The primary endpoint was OS, and there was no selection based on PD-L1 expression. Nivolumab demonstrated a significant improvement in OS compared to chemotherapy in the final analysis [median OS: 10.9 months in the nivolumab group vs. 8.4 months in the chemotherapy group, hazard ratio (HR): $0.77,95 \%$ confidence interval
(CI): 0.62-0.96; $\mathrm{P}=0.019]$. In the subanalysis for OS, the nivolumab group showed a favorable trend regardless of PD-L1 expression [PD-L1 $\geq 1 \%$, HR: 0.69, 95\% CI: 0.51-0.94; PD-L1 <1\%, HR: 0.84, 95\% CI: 0.62-1.14]. The median PFS was 1.7 months and 3.4 months for the nivolumab and chemotherapy groups, respectively, with no statistically significant difference between the two groups (HR: 1.08, 95\% CI: 0.87-1.34). The overall response rates (ORRs) were $19 \%$ in the nivolumab arm and $22 \%$ in the chemotherapy arm, respectively, with a median duration of response of 6.9 months in the nivolumab arm and 3.9 months in the chemotherapy arm. Based on these results, nivolumab was considered the standard of care for second-line treatment of ESCC, regardless of PDL1 expression (9). Pembrolizumab, a humanized IgG4 monoclonal antibody against PD-1, was also studied in a multicohort phase $1 \mathrm{~b}$ global trial (KEYNOTE-028) to evaluate its safety and efficacy in patients with PD-L1positive ( $\geq 1 \%$ of tumor or inflammatory cells or positive stromal band) advanced solid tumors, and it showed promising efficacy in ESCC (10). In the esophageal cancer cohort, 18 of 23 patients had ESCC, and 20 of 23 had received at least two previous chemotherapy regimens. The ORR was $30 \%$ (28\% for ESCC patients). As was seen in clinical trials of nivolumab, the effect on PFS was limited (median PFS: 1.8 months). However, durable responses were observed in some patients; the median OS was 7.0 months, and the 12 -month OS rate was $40 \%$. Further, a phase 2 study (KEYNOTE-180) was performed to assess the efficacy of pembrolizumab for PD-L1-positive SCC or AC of the esophagus or esophagogastric junction (EGJ) in heavily pretreated patients (11). PD-L1 positivity was assessed by immunohistochemistry (PD-L1 IHC 22C3 pharmDx, Agilent Technologies, Carpinteria, CA, USA) and determined using the combined positive score (CPS), which was defined as the number of PD-L1-positive cells (tumor cells, macrophages, and lymphocytes) divided by the total number of tumor cells, multiplied by 100 . One hundred and twenty-one patients were enrolled, of whom $52 \%$ and $48 \%$ had SCC and AC, respectively. Further, $48 \%$ and $52 \%$ of patients had PD-L1-positive (CPS $\geq 10)$ and PD-L1-negative (CPS $<10)$ tumors, respectively. The ORR for the entire population was $9.9 \%$. The SCC, AC, PDL1-positive, and PD-L1-negative subgroups had ORRs of $14.3 \%, 5.2 \%, 13.8 \%$, and $6.3 \%$, respectively. The results indicated that SCC patients and those with PD-L1-positive tumors had much better outcomes with pembrolizumab. The safety profile was consistent with the profile of 
pembrolizumab monotherapy in previous studies. A phase 3 trial (KEYNOTE-181) compared pembrolizumab with the investigator's choice of chemotherapy (paclitaxel, docetaxel, or irinotecan) as second-line treatment in patients with esophageal and EGJ cancer (12). The primary endpoint was OS among patients with PD-L1-positive tumors (CPS $\geq 10$ ), patients with ESCC, and those who intended to undergo treatment. The superiority of pembrolizumab to chemotherapy was demonstrated only in patients with a CPS $\geq 10$. The median OS of patients with a CPS $\geq 10$ in the pembrolizumab group was 9.3 months, whereas it was only 6.7 months in the chemotherapy group (HR: 0.69; $\mathrm{P}=0.0074, \mathrm{P}=0.005$ required). Pembrolizumab also demonstrated an improvement in OS over chemotherapy in ESCC patients, but the difference was not significant (median OS: 8.2 months with pembrolizumab vs. 7.1 months with chemotherapy, HR: 0.78; $\mathrm{P}=0.0095$, required $\mathrm{P} \leq 0.0077$ for significance). These results suggest that pembrolizumab is a therapeutic option for second-line treatment of esophageal cancer patients with a CPS $\geq 10$.

In addition, camrelizumab (SHR-1210), a high-affinity,

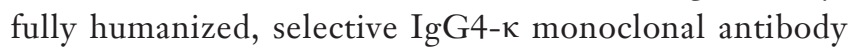
against $\mathrm{PD}-1$, was also studied in a randomized phase 3 trial (ESCORT) to compare with the investigator's choice of chemotherapy (docetaxel or irinotecan) as second-line therapy in patients with advanced or metastatic ESCC, and there was no selection based on PD-L1 expression. Camrelizumab improved OS significantly compared with chemotherapy regardless of PD-L1 expression (median OS: 8.3 months in the camrelizumab group $v s .6 .2$ months in the chemotherapy group, HR: $0.71,95 \%$ CI: $0.57-0.87$; $\mathrm{P}=0.001)$. The camrelizumab group showed a favorable trend regardless of PD-L1 expression in the subanalysis for OS (PD-L1 $\geq 1 \%$, HR: 0.58, 95\% CI: 0.42-0.81; PDL1 <1\%, HR: 0.82 , $95 \%$ CI: $0.62-1.09)$. The median PFS were 1.9 months and 1.9 months for the nivolumab and chemotherapy groups, respectively (HR: 0.69, 95\% CI: $0.56-0.86 ; \mathrm{P}=0.00063)$. The ORRs were $20.2 \%$ in the camrelizumab group and $6.4 \%$ in the chemotherapy group, respectively (13). The results of phase 3 studies of nivolumab and camrelizumab reproducibly showed that PD-1 antibody drugs prolong OS as second-line therapy in patients with ESCC, regardless of PD-L1 expression $(9,13)$.

The clinical trials of nivolumab, pembrolizumab, and camrelizumab in esophageal cancer are summarized in Table 1.

Dual immune checkpoint inhibition and the combination of immunotherapy and cytotoxic agents are also being investigated to achieve increased effectiveness of immunotherapy. In the ESCC cohort of a phase $1 \mathrm{~b}$ study (NCT02658214), the dose-limiting toxicity of the combination of durvalumab (PD-L1 antibody) and tremelimumab (anti-CTLA-4 antibody) in combination with CDDP plus 5-fluorouracil (CF) was the first-line setting (14). Six patients did not experience dose-limiting toxicities with this combination therapy, and two of the six patients had a partial response. A randomized phase 3 trial (ONO-4358-50/CheckMate 648) compared firstline chemotherapy for ESCC patients with nivolumab and ipilimumab (anti-CTLA-4 antibody), CF with nivolumab, or CF (NCT03143153) (15). A randomized phase 3 trial (KEYNOTE-590) compared CF and pembrolizumab with $\mathrm{CF}$ alone as first-line chemotherapy in patients with $\mathrm{AC}$ of the esophagus or EGJ and ESCC (NCT03189719) (16), and this study is ongoing. Further, clinical trials of immune checkpoint inhibitors are underway worldwide, such as trials assessing toripalimab (humanized IgG4 monoclonal anti-PD-1 antibody) (NCT03829969) (17), durvalumab (IgG1-kappa monoclonal anti-PD-L1 antibody) (NCT02962063) (18), SHR1316 (humanized IgG4 monoclonal anti-PD-L1 antibody) (NCT03732508) (19), and tremelimumab (fully human monoclonal anti-CTLA-4 antibody) (NCT03212469) (20). The humanized IgG4 monoclonal anti-PD-1 antibody tislelizumab (BGB-A317) is specifically designed to minimally bind to the Fc receptor on macrophages. A phase 3 trial is underway to compare tislelizumab with other cytotoxic agents (paclitaxel, docetaxel, and irinotecan) as second-line treatment in patients with ESCC (NCT03430843) (21).

\section{irAEs}

irAEs are of increasing interest in clinical practice. irAEs occur when the immune system attempts to restore the immune response by blocking the inhibitory signaling by immune checkpoint inhibitors. Immune checkpoint inhibitors can cause numerous irAEs in various organs, including the skin, lungs, gastrointestinal tract, endocrine system, and nervous system, especially when administered in combination. The presence of diverse irAEs underscores the importance of multidisciplinary care for patients receiving immune checkpoint inhibitors. Clinical guidelines for the management of irAEs have been issued by the American Society of Clinical Oncology and the European Society for Medical Oncology $(22,23)$. It has been reported that the overall incidence of irAEs does not differ based 


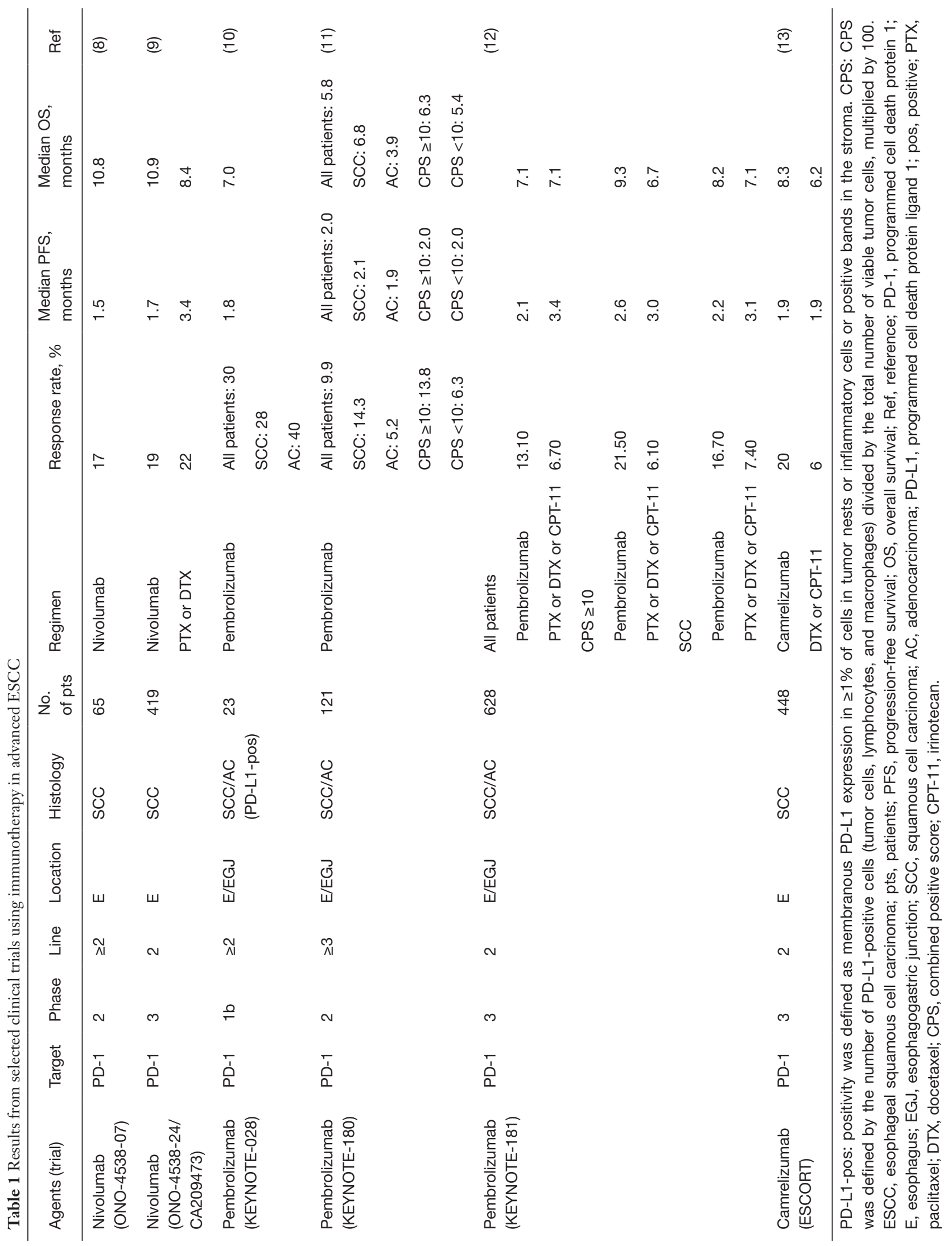


on the primary lesion, but some types of irAEs, such as pneumonitis, have different incidence rates depending on the primary lesion $(24,25)$. In NSCLC, the incidence of pneumonitis is $4 \%$, which is higher than that in other types of cancers (26). Sixty-eight percent of patients treated with camrelizumab received radiotherapy as prior therapy, and the incidence of pneumonitis tended to be as high as $7 \%$ (13). In contrast, $73 \%$ of patients treated with nivolumab had a history of radiation therapy, but the incidence of pneumonitis was only $2 \%$ (9). Because patients with a history of radiotherapy are considered to be at higher risk of pneumonitis, it is necessary to collect and analyze real-world clinical data on the frequency of irAEs, including pneumonitis, in esophageal cancer.

\section{Predictive biomarkers of response}

\section{PD-L1}

The use of the PD-L1 expression level in tumor cells as a predictive biomarker of response is still controversial. In the KEYNOTE-180 trial, patients with a PD-L1 CPS $\geq 10$ had a better prognosis than those with a PD-L1 CPS $<10$ (11). Moreover, the results of the randomized phase 3 KEYNOTE-181 trial showed that pembrolizumab had a significant survival benefit in patients with a PDL1 CPS $\geq 10$ (12). However, the ATTRACTION-3 and ESCORT trials revealed no relationship between clinical efficacy and PD-L1 expression $(9,13)$. However, PD-L1 expression was assessed by tumor proportion score in the ATTRACTION-3 and ESCORT trial, and it is difficult to compare the results with those of KEYNOTE- 181 . Therefore, it remains unclear whether PD-L1 expression levels in esophageal cancer predict the therapeutic efficacy of immunotherapy. Further studies may be necessary.

\section{Tumor mutation burden (TMB)}

TMB refers to the total amount of non-synonymous mutations in the tumor DNA. A previous study showed significant involvement between the TMB and the ORR (27); however, no patients with ESCC were included. Therefore, further studies on ESCC are needed.

\section{High microsatellite instability}

High microsatellite instability is a deficiency of mismatch repair (MMR). MMR is a DNA repair system that corrects
DNA replication errors in normal cells. However, the lack of MMR genes in tumor cells and defects in the process of replication repair increase the likelihood of gene mutations and induce more neoantigen emergence to increase immune cell infiltration (28). This may be why pembrolizumab has shown antitumor effects in MMR-deficient tumors, regardless of the cancer type (29).

Although high microsatellite instability in ESCC is rare, accounting for only about $8 \%$ of cases, this biomarker is essential and may affect the efficacy of immune checkpoint inhibitors (30).

\section{Conclusions}

The results of various clinical trials have shown that immunotherapy is an effective treatment for esophageal cancer. There is no doubt that immunotherapy plays a vital role in the treatment of esophageal cancer, as therapeutic development is progressing worldwide. In addition to its development as palliative treatment, clinical trials have shown its superiority to chemoradiotherapy and its potential as a perioperative treatment, and we expect that more promising treatment options will be developed.

\section{Acknowledgment}

Funding: None.

\section{Footnote}

Reporting Checklist: The authors have completed the Narrative Review reporting checklist. Available at http:// dx.doi.org/10.21037/shc-20-69

Conflicts of Interest: Both authors have completed the ICMJE uniform disclosure form (available at http://dx.doi. org/10.21037/shc-20-69). Dr. KK reports research funds from ONO, MSD, Shionogi, Beigene, Merck Bio, outside the submitted work. The other author has no conflicts of interest to declare.

Ethical Statement: The authors are accountable for all aspects of the work in ensuring that questions related to the accuracy or integrity of any part of the work are appropriately investigated and resolved.

Open Access Statement: This is an Open Access article distributed in accordance with the Creative Commons 
Attribution-NonCommercial-NoDerivs 4.0 International License (CC BY-NC-ND 4.0), which permits the noncommercial replication and distribution of the article with the strict proviso that no changes or edits are made and the original work is properly cited (including links to both the formal publication through the relevant DOI and the license). See: https://creativecommons.org/licenses/by-nc-nd/4.0/.

\section{References}

1. Bray F, Ferlay J, Soerjomataram I, et al. Global cancer statistics 2018: GLOBOCAN estimates of incidence and mortality worldwide for 36 cancers in 185 countries. CA Cancer J Clin 2018;68:394-424.

2. Siewert JR, Ott K. Are squamous and adenocarcinomas of the esophagus the same disease? Semin Radiat Oncol 2007;17:38-44.

3. Sharpe AH, Pauken KE. The diverse functions of the PD1 inhibitory pathway. Nat Rev Immunol 2018;18:153-67.

4. Boussiotis VA. Molecular and biochemical aspects of the PD-1 checkpoint pathway. N Engl J Med 2016; 375:1767-78.

5. Guo W, Wang P, Li N, et al. Prognostic value of PD-L1 in esophageal squamous cell carcinoma: a meta-analysis. Oncotarget 2017;9:13920-33.

6. Page DB, Postow MA, Callahan MK, et al. Immune modulation in cancer with antibodies. Annu Rev Med 2014;65:185-202.

7. Ishida M, Iwai $Y$, Tanaka $Y$, et al. Differential expression of PD-L1 and PD-L2, ligands for an inhibitory receptor PD-1, in the cells of lymphohematopoietic tissues. Immunol Lett 2002;84:57-62.

8. Kudo T, Hamamoto Y, Kato K, et al. Nivolumab treatment for oesophageal squamous-cell carcinoma: an open-label, multicentre, phase 2 trial. Lancet Oncol 2017;18:631-9.

9. Kato K, Cho BC, Takahashi M, et al. Nivolumab versus chemotherapy in patients with advanced oesophageal squamous cell carcinoma refractory or intolerant to previous chemotherapy (ATTRACTION-3): a multicentre, randomised, open-label, phase 3 trial. Lancet Oncol 2019;20:1506-17.

10. Doi T, Piha-Paul SA, Jalal SI, et al. Safety and antitumor activity of the anti-programmed death- 1 antibody pembrolizumab in patients with advanced esophageal carcinoma. J Clin Oncol 2018;36:61-7.

11. Shah MA, Kojima T, Hochhauser D, et al. Efficacy and safety of pembrolizumab for heavily pretreated patients with advanced, metastatic adenocarcinoma or squamous cell carcinoma of the esophagus: the phase 2 KEYNOTE-180 study. JAMA Oncol 2019;5:546-50.

12. Kojima T, Muro K, Francois E, et al. Pembrolizumab versus chemotherapy as second-line therapy for advanced esophageal cancer: Phase III KEYNOTE-181 study. J Clin Oncol 2019;37:2.

13. Huang J, Xu J, Chen Y, et al. Camrelizumab versus investigator's choice of chemotherapy as second-line therapy for advanced or metastatic oesophageal squamous cell carcinoma (ESCORT): a multicentre, randomised, open-label, phase 3 study. Lancet Oncol 2020;21:832-42.

14. Lee DH, Kim H, Keam B, et al. Evaluation of safety and tolerability of durvalumab (D) and tremelimumab (T) in combination with first-line chemotherapy in patients (pts) with esophageal squamous-cell carcinoma (ESCC). J Clin Oncol 2019;37:146.

15. ClinicalTrials.gov. A study to evaluate efficacy in subjects with esophageal cancer treated with nivolumab and ipilimumab or nivolumab combined with fluorouracil plus cisplatin versus fluorouracil plus cisplatin (CheckMate 648). Bethesda: National Library of Medicine (US). Identifier NCT03143153. 2017. (cited 2020 May 22). Available online: https://clinicaltrials.gov/ct2/show/NCT0 3143153 ? cond $=$ NCT $03143153 \&$ draw $=2 \&$ rank $=1$

16. Kato K, Shah MA, Enzinger P, et al. KEYNOTE-590: Phase III study of first-line chemotherapy with or without pembrolizumab for advanced esophageal cancer. Future Oncol 2019;15:1057-66.

17. ClinicalTrials.gov. Toripalimab or placebo with paclitaxel and cisplatin in esophageal squamous cell carcinoma (JUPITER06). Bethesda: National Library of Medicine (US). Identifier NCT03829969. 2019. (cited 2019 Oct 1). Available online: https://clinicaltrials.gov/ct2/show/NCT0 3829969? cond $=$ NCT $03829969 \&$ draw $=2 \&$ rank $=1$

18. ClinicalTrials.gov. Durvalumab, an anti-PD-L1 antibody, and chemoradiation before surgery for esophageal cancer. Bethesda: National Library of Medicine (US). Identifier NCT02962063. 2016. (cited 2019 Jun 5). Available online: https://clinicaltrials.gov/ct2/show/NCT02962063? cond= NCT02962063\&draw $=2 \&$ rank $=1$

19. ClinicalTrials.gov. SHR-1316 in combination with chemotherapy in patients with esophageal squamous cell cancer. Bethesda: National Library of Medicine (US). Identifier NCT03732508. 2018. (cited 2018 Nov 6). Available online: https://clinicaltrials.gov/ct2/show/NCT0 3732508 ? cond $=$ NCT03732508 $\&$ draw $=2 \&$ rank $=1$

20. ClinicalTrials.gov. A trial of durvalumab and 
tremelimumab in comibination with SBRT in patients with metastatic cancer (ABBIMUNE). Bethesda: National Library of Medicine (US). Identifier NCT03212469. 2017. (cited 2018 Jul 24). Available online: https://clinicaltrials. gov/ct2/show/NCT03212469? cond=NCT03212469\&dra $\mathrm{w}=2 \& \operatorname{rank}=1$

21. ClinicalTrials.gov. A study of tislelizumab (BGB-A317) versus chemotherapy as second line treatment in participants with advanced esophageal squamous cell carcinoma. Bethesda: National Library of Medicine (US). Identifier NCT03430843. 2018. (cited 2020 Mar 19). Available online: https://clinicaltrials.gov/ct2/show/NCT0 3430843 ? cond $=$ NCT03430843 \&draw $=2 \&$ rank $=1$

22. Brahmer JR, Lacchetti C, Schneider BJ, et al. Management of immune-related adverse events in patients treated with immune checkpoint inhibitor therapy: American society of clinical oncology clinical practice guideline. J Clin Oncol 2018;36:1714-68.

23. Haanen JBAG, Carbonnel F, Robert C, et al. Management of toxicities from immunotherapy: ESMO Clinical Practice Guidelines for diagnosis, treatment and followup. Ann Oncol 2018; 29:iv264-6.

24. Khoja L, Day D, Wei-Wu Chen T, et al. Tumour- and class-specific patterns of immune-related adverse events of

doi: $10.21037 /$ shc-20-69

Cite this article as: Miyamoto T, Kato K. Immunotherapy for esophageal carcinoma: a narrative review. Shanghai Chest 2021;5:26. immune checkpoint inhibitors: a systematic review. Ann Oncol 2017;28:2377-85.

25. Wang Y, Zhou S, Yang F, et al. Treatment-related adverse events of PD-1 and PD-L1 inhibitors in clinical trials: a systematic review and meta-analysis. JAMA Oncol 2019;5:1008-19.

26. Sun X, Roudi R, Dai T, et al. Immune-related adverse events associated with programmed cell death protein-1 and programmed cell death ligand 1 inhibitors for nonsmall cell lung cancer: a PRISMA systematic review and meta-analysis. BMC Cancer 2019;19:558.

27. Yarchoan M, Hopkins A, Jaffee EM. Tumor mutational burden and response rate to PD-1 inhibition. N Engl J Med 2017;377:2500-1.

28. Boland CR, Goel A. Microsatellite instability in colorectal cancer. Gastroenterology 2010;138:2073-87.e3.

29. Le DT, Durham JN, Smith KN, et al. Mismatch repair deficiency predicts response of solid tumors to PD-1 blockade. Science 2017;357:409-13.

30. Matsumoto Y, Nagasaka T, Kambara T, et al. Microsatellite instability and clinicopathological features in esophageal squamous cell cancer. Oncol Rep 2007;18:1123-7. 